The Astrophysical Journal, 296:379-389, 1985 September 15

(C) 1985. The American Astronomical Society. All rights reserved. Printed in U.S.A.

\title{
TYPE I SUPERNOVAE IN THE INFRARED AND THEIR USE AS DISTANCE INDICATORS
}

\author{
J. H. Elias, ${ }^{1}$ K. Matthews, ${ }^{1}$ G. Neugebauer, ${ }^{1}$ and S. E. Persson ${ }^{2}$ \\ Received 1985 January 28; accepted 1985 March 22
}

\begin{abstract}
New infrared data for 11 Type I supernovae are presented. These results, when combined with other published data for Type I supernovae, show that the light curves fall into two well-defined groups. The first, more common type-Type Ia-shows strong, variable, unexplained absorption at $1.2 \mu \mathrm{m}$ and probably at 3.5 $\mu \mathrm{m}$, while the second type-Type Ib-shows no such absorption and a slower decline after maximum. The light curves of the Type Ia supernovae appear to have a dispersion in color and absolute magnitude of \pm 0.2 mag or less, making them potentially valuable for distance determination within the Local Supercluster.

Subject headings: cosmology — infrared: sources — stars: supernovae
\end{abstract}

\section{INTRODUCTION}

Type I supernovae are the most luminous stellar objects known, so it is natural for them to be considered for use as distance indicators. In visible light, their light curves show a dispersion in magnitude at maximum light of a few tenths of a magnitude. It is a matter of dispute (cf. Barbon 1980; Sandage and Tammann 1982) whether this dispersion is due primarily to differences in the interstellar extinction in the parent galaxies, or is an intrinsic dispersion in the magnitudes of the supernovae themselves, which may be correlated with other properties of the light curves.

In the near-infrared, interstellar extinction is several times less than in the visible, and other effects (e.g., temperature) might also lead to smaller dispersion. Observations of three Type I supernovae (two in NGC 1316 and one in NGC 4536) (Elias et al. 1981, hereafter Paper I), combined with observations of SN 1972e in NGC 5253 by Kirshner et al. (1973b) and Lee et al. (1972) indicated that the dispersion in absolute magnitude was \pm 0.2 mag or less.

In this paper, we present initial results of a program aimed at enlarging the sample of Type I supernovae with infrared data. A total of seven Type I supernovae have been observed (see Table 2). Other, older observations of SN 1971 i in NGC 5055, of SN 19711 in NGC 6384, and some additional observations of SN $1981 b$ in NGC 4536 are also given. Observations of the supernovae in M83 and NGC 4419 have been published by Meikle et al. (1985), Meikle, Graham, and Bode (1984), Meikle and Walther (1984), and observations of the supernova in NGC 991 have been published by Graham et al. (1984); there are thus 13 Type I supernovae in 12 galaxies which have been observed in the infrared. These data are used to show that Type I supernovae separate into two groups in the infrared, based on their colors, and to examine the utility of the first group - Type Ia-as distance indicators.

In addition, examination of the 3.5 and $3.7 \mu \mathrm{m}$ data for Type Ia supernovae suggests that their energy distribution are depressed at $\sim 3.5 \mu \mathrm{m}$ by an amount nearly comparable to the depression at $1.2 \mu \mathrm{m}$. Examination of the visual-infrared colors and comparison with visual colors indicates that some caution must be used in deducing supernova effective temperatures from any one color; a blackbody fit, such as that used by

\footnotetext{
${ }^{1}$ Palomar Observatory, California Institute of Technology.

${ }^{2}$ Mount Wilson and Las Campanas Observatories.
}

Kirshner et al. (1973b) and Branch et al. (1983), appears preferable. There appears to be scatter in the $B-V$ color curves, when compared with other color curves or with spectroscopic phases for the supernovae, suggesting that phases (time of maximum) deduced from $B-V$ colors alone are unreliable.

\section{OBSERVATIONS AND RESULTS}

\section{a) Infrared Photometry}

The observing program consists in principle of all accessible Type I supernovae occurring in galaxies of redshift $\$ 3000 \mathrm{~km}$ $\mathrm{s}^{-1}$. Supernovae which could not be observed within a month of maximum light were not observed. Relevant data for the supernovae and their parent galaxies are listed in Table 1. The supernovae in NGC 1316, 4536, and 5253 are also included. Four other objects have been observed: the Type II supernova in NGC 3169, results on which will be reported elsewhere; a supernova of unknown type in IC 121 and a possible supernova in NGC 7184, for both of which results are given in Table 2; and the "supernova" in NGC 6217 (Doroshenko and Tsvetkov 1983), which is in fact a foreground G star (R. P. Kirshner, private communication; our photometry of $K=13.75$, $H-K=0.11$, and $J-H=0.34$, supports this identification).

All but one of the measurements were obtained using the 5 $\mathrm{m}$ Hale Telescope at Palomar Observatory with an InSb detector. One measurement of SN 19841 in NGC 991 was made on the CTIO $4 \mathrm{~m}$ telescope. ${ }^{3}$ Observations were usually made with a $5^{\prime \prime}$ beam diameter and chopper spacing ranging from $5^{\prime \prime}$ to $15^{\prime \prime}$. Careful measurements were made to determine corrections for the effects of the underlying galaxy on the supernova measurements. In two cases, NGC 4051 and NGC 4753, a measurement was made at the position of the supernova roughly a year after maximum light in order to verify the accuracy of the corrections. The infrared photometry is presented in Table 2. The values include the corrections for the parent galaxy, and the tabulated uncertainties include the estimated uncertainties in the corrections. Near maximum light, the corrections are usually less than $0.1 \mathrm{mag}$ at all wavelengths. Some remarks on measurements of individual supernovae are given below. Previously unpublished measurements for SN 1981b in NGC 4536 are also given in Table 2.

\footnotetext{
${ }^{3}$ Cerro Tololo Inter-American Observatory-National Optical Astronomy Observatories is operated by AURA, Inc., under contract with the National Science Foundation.
} 
TABLE 1

Supernova and Parent Galaxy Properties

\begin{tabular}{|c|c|c|c|c|c|c|c|c|c|}
\hline Galaxy & $V_{0}\left(\mathrm{~km} \mathrm{~s}^{-1}\right)$ & $D / D_{\mathrm{Vir}^{\mathrm{a}}}^{\mathrm{a}}$ & $A_{B}(\mathrm{mag})^{\mathrm{b}}$ & $\mathrm{SN}$ & $t_{\max }(\mathrm{JD})^{\mathrm{c}}$ & $t_{0}(\mathrm{JD})^{\mathrm{c}}$ & $H_{20}$ & $\chi^{2}$ & $n^{\mathrm{d}}$ \\
\hline IC $121 \ldots \ldots$. & 9114 & 6.73 & +0.04 & $1984 \mathrm{~m}$ & & & & & $\ldots$ \\
\hline NGC $991 \ldots$ & 1533 & 1.077 & +0.00 & 19841 & (5943) & $\ldots$ & $\ldots$ & $\ldots$ & $\ldots$ \\
\hline IC $1731 \ldots \ldots$ & 3590 & 2.623 & +0.22 & $1983 r$ & $(5602)$ & $5608.2(10)$ & $15.03(7)$ & 1.3 & 6 \\
\hline \multirow[t]{2}{*}{ NGC 1316 .. } & 1658 & 1.203 & -0.02 & $1980 n$ & 4586. & $4580.2(2)$ & $13.31(2)$ & 18.2 & 25 \\
\hline & $\ldots$ & $\ldots$ & $\ldots$ & $1981 d$ & 4680 . & $4672.7(2)$ & $13.35(2)$ & 13.8 & 14 \\
\hline NGC 3227 .. & 1098 & 1.234 & +0.02 & $1983 u$ & $(\leq 5643)$ & $5642.5(10)$ & $13.66(9)$ & 0 & 2 \\
\hline NGC $4051 \ldots$ & 763 & 0.623 & -0.06 & $1983 \mathrm{i}$ & (5443) & $\ldots$ & .. & $\ldots$ & $\ldots$ \\
\hline NGC 4419 .. & -251 & 1.000 & +0.06 & $1984 a$ & $(5710)$ & $5713.6(10)$ & $13.28(6)$ & 34: & 10 \\
\hline NGC 4536 .. & 1682 & $0.84:$ & -0.02 & $1981 b$ & 4672.5 & $4667.3(3)$ & $12.92(2)$ & 39.2 & 26 \\
\hline NGC $4753 \ldots$ & 1169 & 1.00: & +0.02 & $1983 \mathrm{~g}$ & $(5422)$ & $5429.4(5)$ & $13.67(3)$ & 16.6 & 10 \\
\hline NGC 5055 .. & 575 & 0.415 & -0.12 & $1971 \mathrm{i}$ & 1100.5 & (1095) & $12.87(30)$ & 1.2 & 3 \\
\hline NGC $5236 \ldots$ & 339 & 0.275 & +0.14 & $1983 n$ & $(5528)$ & $\ldots$ & $\ldots$ & $\ldots$ & $\ldots$ \\
\hline NGC 5253 .. & 211 & 0.169 & +0.19 & $1972 \mathrm{e}$ & 1447.5 & $1444.5(9)$ & $9.35(4)$ & 14.9 & 14 \\
\hline NGC 6384 .. & 1735 & 1.492 & +0.41 & 19711 & 1131.5 & (1126) & $13.64(32)$ & 0 & 1 \\
\hline
\end{tabular}

${ }^{a}$ Distance in units of distance to Virgo Cluster center, based on infall model of Aaronson et al. 1982. See text for details.

${ }^{\mathrm{b}}$ Foreground extinction at $B$ from Burstein and Heiles 1984. Negative values are treated as zero. Corrections were done using $A_{K}=0.066 A_{B}, A_{H}=0.112 A_{B}, E_{J-H}=0.083 A_{B}$, and $E_{H-K}=0.046 A_{B}$.

${ }^{c}$ Dates are Julian Date minus 2,440,000. Values of $t_{\max }$ are estimates of time of maximum blue light. Numbers without parentheses are directly observed maxima; values in parentheses are indirect determinations. Values of $t_{0}$ are phase determined from infrared data, followed by uncertainties in 0.1 in parentheses. Values for SN 19711 and $1971 \mathrm{i}$ are adopted values. See text for further details.

${ }^{d}$ Number of $H$ and $J-H$ values used in fitting $t_{0}$ and $H_{20}$. For SN 1971i and SN 19711, $n$ is the number of points at $K$.

\section{i) NGC 4419 (SN 1984a) and NGC 991 (SN 1984l)}

Other infrared measurements were done by Meikle, Graham, and Bode (1984), Meikle and Walther (1984), and Graham et al. (1984). The galaxy correction to the Palomar measurement of SN 1984a is large since the supernova was faint; corrections of comparable size will affect the data of Meikle and colleagues by 0.1 mag or less.

$$
\text { ii) } N G C 5236(M 83)(S N \text { 1983n) }
$$

Earlier measurements, including some made at Palomar, are given in Meikle et al. (1985). The data in Table 2 are subsequent observations. The uncertainties include the uncertainty in the atmospheric extinction corrections for this southern object.

\section{iii) NGC 5055 (SN 1971i) and NGC 6384 (SN 1971l)}

Observations of the supernovae were made in 1971 at $K$ only. These older measurements are of distinctly lower precision than those of the other objects in Table 2. NGC 5055 is generally thought to be a member of the M101 group, (e.g., de Vaucouleurs 1975), so this supernova may be important for distance determinations.

$$
\text { iv) } N G C 5253=S N 1972 e
$$

Observations of this supernova were published in graphic form by Kirshner et al. (1973b). In the Appendix these data are presented in tabular form, together with further, unpublished data from 1973-1975. The data are of variable quality: the measurements on the larger telescopes are of quality comparable to that of the modern photometry in Table 2, while other measurements are of lower accuracy.

$$
\text { v) } N G C 7184=S N 1984 \text { ? and IC } 121=\text { SN } 1984 n
$$

No spectroscopic classification is available for either of these two supernovae. The colors and lack of variability of the object measured in NGC 7184 indicate that it is probably a foreground star (probably an F dwarf). Whether another object in NGC 7184 was a supernova is unclear (Marsden 1984, and references therein). The star measured in NGC 7184 was $69^{\prime \prime}$ north and $54^{\prime \prime}$ east of the galaxy nucleus.

\section{b) Light Curves}

The data in Paper I define composite light curves with little scatter for roughly the first 60 days after maximum light. A reasonable approximation to these are the fiducial color and light curves in $J-H, H-K$, and $H$ which are tabulated in Table 3.

The fiducial curves were produced by fitting the data points for the three supernovae from Paper I to curves made up of a series of straight line segments. The data in the present paper were then added, resulting in small changes in the fiducial curves. The choice of the times at which the curves are defined was somewhat arbitrary, though not of critical importance. The choice of a small number of defining points for the fiducial curves, as opposed to "smoother" curves or analytical approximations, simplifies presentation of the curves and also reflects our view of the limits of the data. The fiducial curves are presented in this explicit, tabular form in order to enable others to make use of them for new supernova data.

The uncertainties in the fiducial curves are roughly \pm 0.03 mag between $t=5$ days and $t=40$ days. Earlier than this, there are no data, and later than $t=40$ days the data are sparser and of lower accuracy, so that by $t=100$ days, the uncertainties in the fiducial curves may be as great as \pm 0.1 mag. For this reason, data later than $t=100$ days were not used in the fits described below.

In constructing the fiducial curves and in fitting the new data to the curves, corrections were applied for foreground reddening, using the extinction values and reddening coefficients noted in Table 1 . These corrections are generally small, $0.02 \mathrm{mag}$ or less.

The fiducial curves were used to fit the data for the 11 supernovae with data at $J, H$, and $K$. All of the data were corrected for foreground extinction, using the values given in Table 1. In fitting the data, there are two parameters to be determined: the phase, defined by an approximate time of maximum $\left(t_{0}\right.$ in Table 1) and the overall level of the $H$ curve ( $H$ at $t=20$ days $\left[H_{20}\right]$ in Table 2). The best-fit values for $t=0$ and $H_{20}$ for the eight other supernovae are listed in Table 1, together with $n$, 
TABLE 2

SUPERNOVA PHOTOMETRY

\begin{tabular}{cccccc}
\hline \multicolumn{7}{c}{ Galaxy } & Date $^{\mathrm{a}}$ & $J$ & $H$ & $K$ & Correction $^{\mathrm{b}}$ \\
\hline \multicolumn{6}{c}{ SN 1971i } \\
\hline NGC 5055 $\ldots$ & 1122.8 & $\ldots$ & $\ldots$ & $12.99(47)$ & $\mathrm{x}$ \\
& 1132.8 & $\ldots$ & $\ldots$ & $13.06(13)$ & $\mathrm{x}$ \\
& 1156.8 & $\ldots$ & $\ldots$ & $14.46(32)$ & $\mathrm{x}$ \\
\hline
\end{tabular}

\begin{tabular}{|c|c|c|c|c|c|}
\hline \multicolumn{6}{|c|}{ SN 19711} \\
\hline NGC 6384 . & 1132.8 & $\ldots$ & $\ldots$ & $13.40(19)$ & $\mathrm{x}$ \\
\hline \multicolumn{6}{|c|}{ SN $1981 b^{c}$} \\
\hline NGC $4536 \ldots$ & $\begin{array}{l}4679.0 \\
4737.7\end{array}$ & $\begin{array}{l}13.19(7) \\
16.09(15)\end{array}$ & $\begin{array}{l}12.95(6) \\
14.53(7)\end{array}$ & $\begin{array}{l}12.81(5) \\
14.67(7)\end{array}$ & $\begin{array}{l}1 \\
1\end{array}$ \\
\hline \multicolumn{6}{|c|}{ SN 1983g } \\
\hline NGC $4753 \ldots$ & $\begin{array}{l}5448.7^{\mathrm{d}} \\
5476.7 \\
5479.7 \\
5493.8 \\
5495.7\end{array}$ & $\begin{array}{l}14.98(6) \\
15.35(6) \\
15.51(6) \\
16.59(16) \\
16.19(9)\end{array}$ & $\begin{array}{l}13.69(4) \\
14.37(4) \\
14.43(3) \\
15.16(7) \\
15.14(13)\end{array}$ & $\begin{array}{l}13.61(4) \\
14.42(5) \\
14.56(5) \\
15.18(8) \\
15.32(11)\end{array}$ & $\begin{array}{l}2 \\
3 \\
3 \\
4 \\
3\end{array}$ \\
\hline
\end{tabular}

\begin{tabular}{llllll}
\hline \multicolumn{6}{c}{ SN 1983i } \\
\hline NGC 4051 $\ldots$ & 5493.8 & $14.39(11)$ & $13.93(7)$ & $13.90(5)$ & 2 \\
& 5496.6 & $14.47(6)$ & $13.97(4)$ & $13.96(3)$ & 2 \\
& 5538.7 & $15.91(6)$ & $15.15(4)$ & $15.16(7)$ & 3 \\
& 5539.7 & $15.80(8)$ & $15.08(5)$ & $15.13(5)$ & 2 \\
& 5543.7 & $16.10(7)$ & $15.22(5)$ & $15.20(6)$ & 3 \\
\hline
\end{tabular}

\begin{tabular}{llllll}
\hline \multicolumn{6}{c}{ SN 1983n } \\
\hline NGC 5236 $\ldots$ & 5738.0 & $15.44(7)$ & $14.87(7)$ & $14.58(8)$ & 3 \\
& 5807.8 & $15.98(8)$ & $15.48(8)$ & $15.78(14)$ & 4 \\
& 5829.8 & $16.86(13)$ & $16.40(15)$ & $16.77(44)$ & 4 \\
& 5862.7 & $16.91(22)$ & $16.27(18)$ & $17.52(94)$ & 4 \\
\hline
\end{tabular}

\begin{tabular}{llllll}
\hline \multicolumn{5}{c}{ SN 1983r } \\
\hline IC $1731 \ldots \ldots$ & 5626.8 & $16.23(15)$ & $15.05(10)$ & $15.11(13)$ & 4 \\
& 5631.9 & $16.18(18)$ & $15.01(12)$ & $14.88(12)$ & 4 \\
& 5658.7 & $17.07(11)$ & $15.88(8)$ & $15.92(9)$ & 2 \\
\hline
\end{tabular}

\begin{tabular}{cccccc}
\hline \multicolumn{6}{c}{ SN 1983u } \\
\hline NGC $3227 \ldots$ & 5661.1 & $14.93(9)$ & $13.69(7)$ & $13.74(6)$ & 2 \\
& $5806.8^{\mathrm{f}}$ & $\ldots$ & $\ldots$ & $\ldots$ & 4 \\
\hline
\end{tabular}

\begin{tabular}{|c|c|c|c|c|c|}
\hline \multicolumn{6}{|c|}{ SN $1984 a^{8}$} \\
\hline NGC $4419 \ldots$ & 5807.9 & $18.06(30)$ & $16.12(12)$ & $16.11(13)$ & 4 \\
\hline \multicolumn{6}{|c|}{ SN 1984? } \\
\hline \multirow[t]{2}{*}{ NGC $7184 \ldots$} & 5941.8 & $14.01(5)$ & $13.83(5)$ & $13.74(5)$ & $\mathrm{x}$ \\
\hline & 5979.6 & $14.16(4)$ & $13.97(4)$ & $13.85(4)$ & $\mathrm{x}$ \\
\hline \multicolumn{6}{|c|}{ SN $1984^{\mathrm{h}}$} \\
\hline \multirow[t]{5}{*}{ NGC $991 \ldots$} & $5959.7^{i}$ & $13.26(9)$ & $13.07(8)$ & $12.87(7)$ & 1 \\
\hline & 5979.7 & $13.63(4)$ & $13.32(4)$ & $13.20(4)$ & 1 \\
\hline & 6008.6 & $14.49(6)$ & $14.00(4)$ & & 1 \\
\hline & 6009.6 & $14.42(4)$ & $13.91(4)$ & $13.97(4)$ & 1 \\
\hline & $6050.6^{\mathrm{j}}$ & $15.62(8)$ & $14.88(5)$ & $15.05(4)$ & 1 \\
\hline \multicolumn{6}{|c|}{ SN $1984 \mathrm{~m}$} \\
\hline IC $121 \ldots \ldots$ & 5959.7 & $17.77(25)$ & $17.21(28)$ & & $\mathrm{x}$ \\
\hline & 5979.7 & $18.52(21)$ & $18.85(14)$ & $18.52(110)$ & $\mathrm{x}$ \\
\hline
\end{tabular}

the number of points at $J-H$ and $H$ used in the fit and the resulting $\chi^{2}$. The $H-K$ data were not used in fitting because the $H-K$ color varies so little; the data would match the fiducial light curve well for any plausible choice of $t_{0}$. In principle, one could also determine the reddening due to the parent galaxy, but this appears to be less than 0.05 mag at $H$ for all the supernovae considered and could not be measured with any significance for any object.

Some examples of the fits are shown in Figures 1-3. Figure 1 shows the data for the three supernovae from Paper I, including the additional data from Table 2. Figure 2 shows the data for the four Type Ia supernovae (see discussion below) observed in 1983/1984. Figure 3 shows the data for SN 1972e. All the data in these figures have been corrected for foreground extinction and shifted using the values of $t_{0}$ and $H_{20}$ given in Table 1. While most of the supernovae fit the fiducial curves well, three do not. SN 1983n in M83, SN 19841 in NGC 991, and SN 1983i in NGC 4051 show bluer $J-H$ colors and a slower decline at $H$ than the fiducial curves (Figs. 4 and 5).

In Table 1 values for the time of maximum blue light (" $t_{\max }$ ") determined from photometry or spectroscopy are listed. Values in parentheses are for supernovae where time of maximum was inferred from spectroscopic data or visual colors. There are only four objects where $t_{0}$ is determined and maximum light has been observed in the visual. For these three supernovae, $t_{\max }-t_{0}=5.3 \pm 0.9$ days. For the remaining objects, the time of maximum was determined indirectly, either from spectra or $B-V$ color curves. If these three objects are included, one gets $t_{\max }-t_{0}=+0.6 \pm 2.3$ days. This is significantly different from the value determined from directly observed maximum; the discrepancy is discussed below.

The two 1971 supernovae were observed at visual wavelengths at maximum light, so $t_{0}$ was taken to be 5.5 days earlier in each case in order to determine $H_{20}$; these adopted values are given in parenthesis in Table 1. The data for these two objects are at $K$ only, so a $K$ fiducial curve was fitted to the data to arrive at values of $H_{20}$. The uncertainties in the values of $H_{20}$ include the effects of an assumed 5 day uncertainty in $t_{0}$. This contribution to the uncertainty is about $0.25 \mathrm{mag}$ in both cases. If one of the supernovae is not Type Ia then the estimates of its $\mathrm{H}_{20}$ will of course be incorrect.

For SN 1984a in NGC 4419, values for $t_{0}$ and for $H_{20}$ were determined applying a range of corrections for the underlying galaxy to the Meikle, Graham, and Bode (1984) and Meikle and Walther (1984) data. Since this supernova lies in a spiral

\footnotetext{
a Date is Julian Day $-2,440,000$.

b The size of the correction for the underlying galaxy is indicated by the following code: $(1)<2 \%$ at all wavelengths; $(2)$ greater than $2 \%$ at least one wavelength but less than $10 \%$ at all wavelengths; (3) greater than $10 \%$ at least one wavelength, but less than $30 \%$ at all wavelengths; $(4)$ greater than $30 \%$ at least one wavelength; and (x) unknown, believed to be smaller than the tabulated uncertainties in the flux. Tabulated magnitudes include these corrections. See text.

c Additional observations of SN 1981 b published by Elias et al. 1981.

${ }^{d}$ Measurement at $L^{\prime}(3.7 \mu \mathrm{m})$ on this date gives $L^{\prime}=14.16(47)$.

c Additional observations of SN 1983n, including 1983 Palomar measurements, are given in Meikle et al. 1985.

$\mathrm{f}$ Corrections were very large relative to supernova on this date; an upper limit to the supernova is $H>17.5$.

${ }^{g}$ Additional observations of SN 1984a published by Meikle et al. 1984a, $b$

${ }^{\mathrm{h}}$ Additional observations of SN 19841 published by Graham et al. 1984.

' Measurement at $L^{\prime}(3.7 \mu \mathrm{m})$ on this date gives $L^{\prime}=12.66(43)$.

j Measurement made on CTIO $4 \mathrm{~m}$ telescope.
} 
TABLE 3

Fiducial Light Curves

\begin{tabular}{cccc}
\hline \hline $\begin{array}{c}t-t_{0} \\
\text { (days) }\end{array}$ & $J-H^{\mathrm{a}}$ & $H-K^{\mathrm{a}}$ & $H-H_{20}{ }^{\mathrm{a}, \mathrm{b}}$ \\
\hline $0 \ldots \ldots$ & $-0.5:$ & $+0.5:$ & $-0.13:$ \\
$10 \ldots \ldots$ & -0.18 & +0.17 & +0.07 \\
$15 \ldots \ldots$ & +0.73 & -0.01 & +0.20 \\
$20 \ldots \ldots$ & +1.33 & +0.03 & +0.00 \\
$23 \ldots \ldots$ & +1.31 & +0.05 & -0.13 \\
$30 \ldots \ldots$ & +0.95 & -0.11 & -0.36 \\
$38 \ldots \ldots$ & +0.66 & -0.14 & +0.13 \\
$40 \ldots \ldots$ & +0.76 & -0.16 & +0.24 \\
$50 \ldots \ldots$ & +1.16 & -0.13 & +0.82 \\
$60 \ldots \ldots$ & +1.36 & -0.13 & +1.22 \\
$70 \ldots \ldots$ & +1.60 & -0.12 & +1.59 \\
$80 \ldots \ldots$ & +1.65 & -0.05 & +2.04 \\
$100 \ldots \ldots$ & $+1.6:$ & +0.05 & +2.92 \\
$120 \ldots \ldots$ & $+1.6:$ & $+0.2:$ & $+3.5:$ \\
\hline
\end{tabular}

${ }^{a}$ Magnitudes and colors after correction for Galactic foreground absorption.

${ }^{\mathrm{b}}$ Magnitude relative to magnitude at $t-t_{0}=20^{\mathrm{d}}$.

arm, the true supernova magnitudes are likely to be fainter than the observed values. The corrections determined for the Palomar data are in this sense; the corrections applied to the data of Meikle and colleagues varied between 0 and 5 times the Palomar corrections; the resulting range in $t_{0}$ is less than 1 day, and the range in $H_{20}$ is $0.1 \mathrm{mag}$.

\section{c) Type Ib Supernovae}

The three Type Ib supernovae, SN 1983i, SN 1983n, and SN

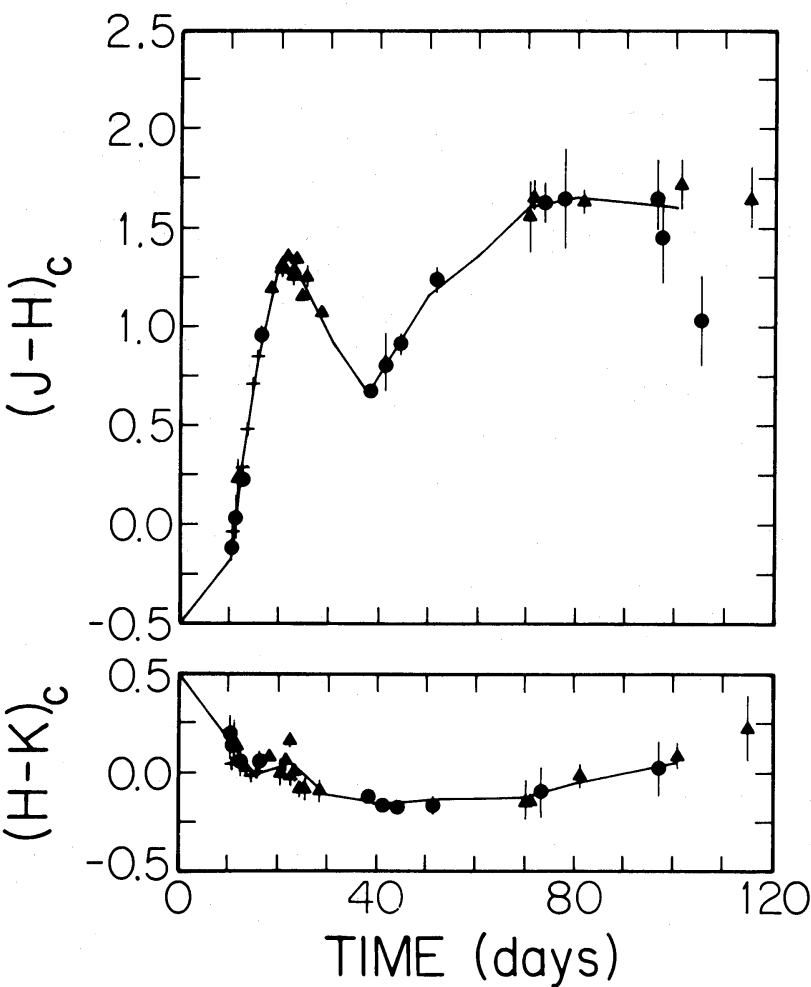

19841 appear to have similar light curves. ${ }^{4}$ Since rough estimates of $t_{\max }$ are available (Table 1), composite color and light curves can be constructed (Figs. 4 and 5). The data for SN 1983 i have been shifted by 85 days to match the SN 1983n data, and the data for SN 19841 have been shifted by 415 days. The evolution in color and in magnitude is relatively slow, so uncertainties in the relative times of maximum light do not affect the detailed matchup of the curves. In order to fit the $H$ light curve, the SN 1983 i data must be made brighter by 3.0 mag, and the SN 19841 data must be made brighter by 2.7 mag. A change of 5 days in the relative time of maximum light would change the best-fit magnitude shifts by 0.2 mag or less. Note that the distances given in Table 1 imply that SN $1983 \mathrm{i}$ in NGC 4051 is fainter than the other two Type Ib supernovae by $\sim 1 \mathrm{mag}$ in absolute magnitude.

Comparison of the composite color and light curves in Figure 4 with the curves for normal Type I supernovae in Figures $1-3$ shows differences. First, $J-H$ is bluer in the peculiar supernovae than in the normal Type I supernovae after the first few days, and varies slowly, rising gradually to a value of 0.6. The $H-K$ curves look similar for both types, but it appears that the Type Ia supernovae are somewhat bluer by $\sim 0.1 \mathrm{mag}$ from about $t=20$ days to $t=60$ days. Finally, the $H$ light curve peaks soon after visual maximum and shows no sign of a second maximum near $t=30$ days. The decline after

\footnotetext{
${ }^{4}$ Note added in proof.-Spectroscopic observations in the visual of two of the Type Ib supernovae, SN 19841 and SN 1983n, have shown that there are in fact well-defined differences between their spectra and those of Type Ia supernovae (e.g. Wheeler and Levreault 1985 and references therein).
}

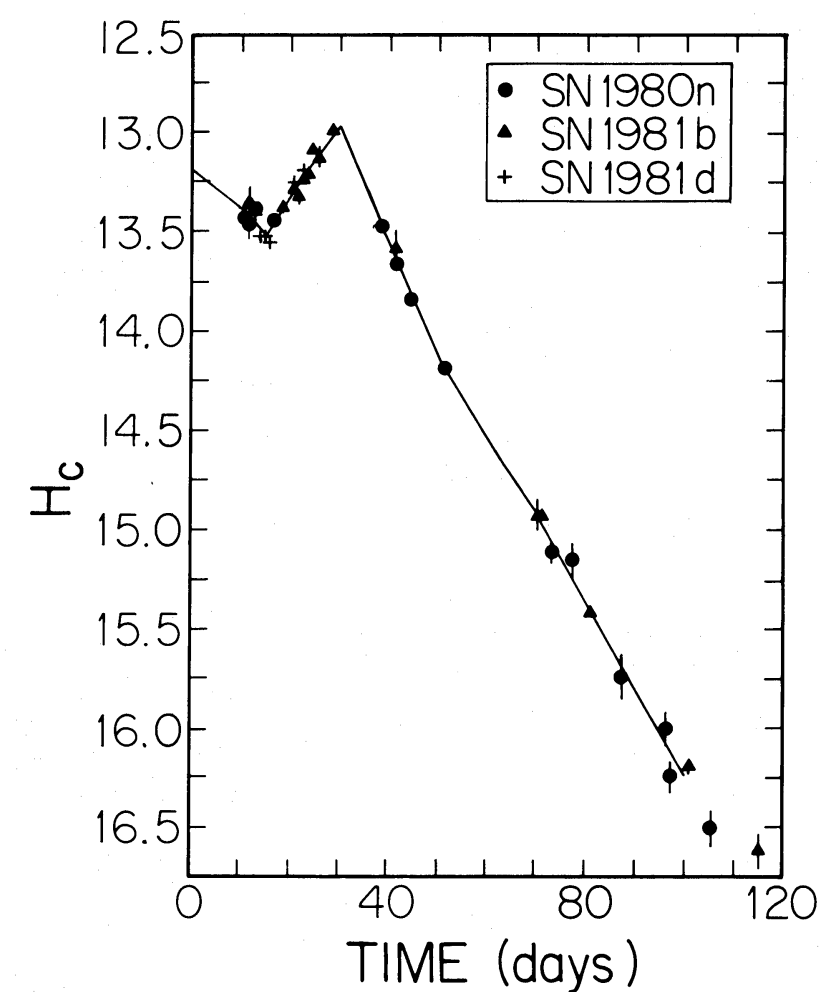

Fig. 1. $-J-H, H-K$, and $H$ light curves for SN 1980n, SN 1981b, and SN 1981d matched to the fiducial curves from Table 3. All data from Table 2 or Paper I. The data have been corrected for foreground reddening (see text for details). The $H$ data for SN 1981b and SN 1981d have been shifted to conform to the SN $1980 \mathrm{n} H$ magnitude scale; the shifts are given by the differences in $H_{20}$ values given in Table 1 . 

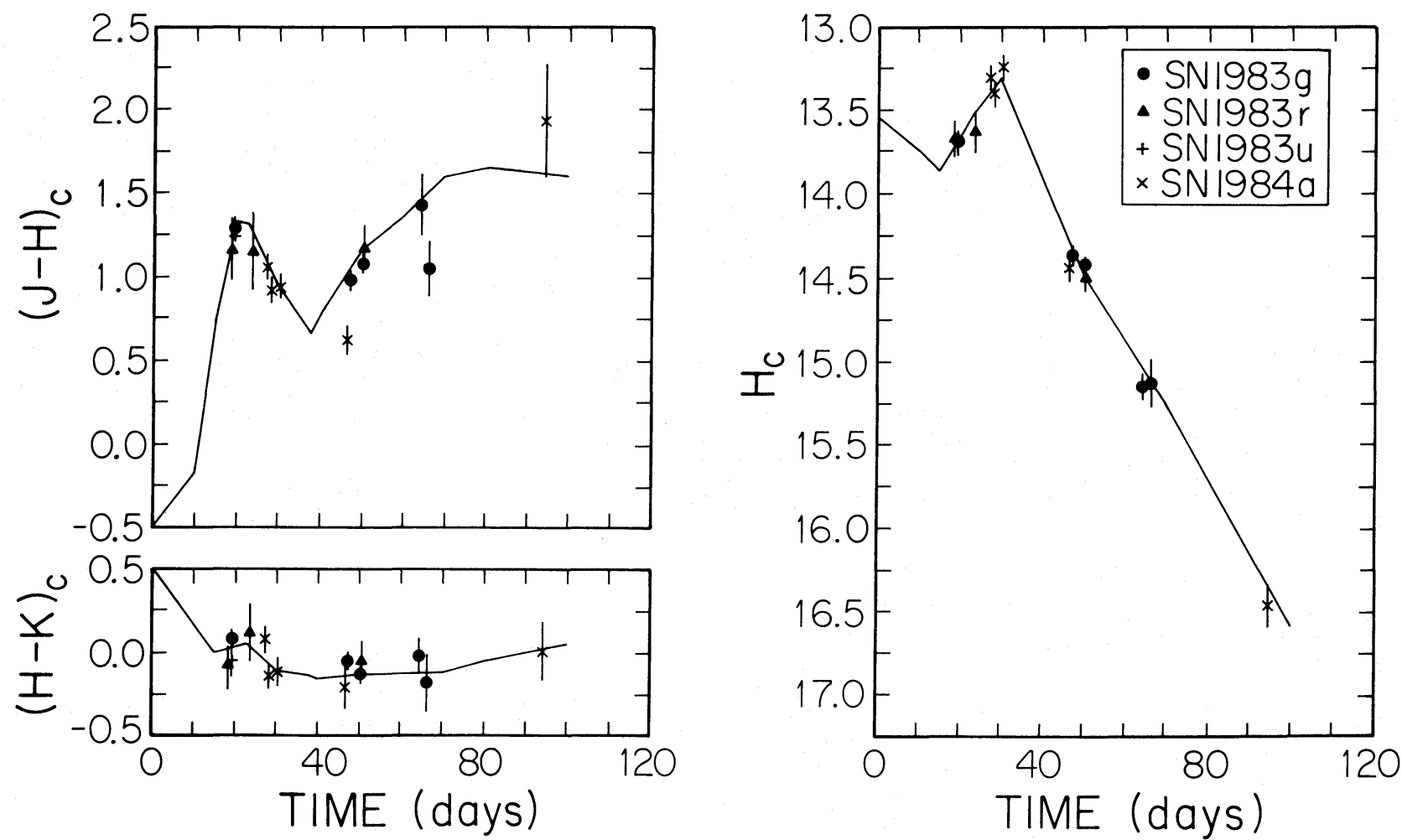

Fig. 2.- Infrared data for SN 1983g, SN 1983r, SN 1983u, and SN 1984a plotted as in Fig. 1. The $H$ magnitude scale shown is for the SN 1983g data; data for the other supernovae have been shifted as in Fig. 1.
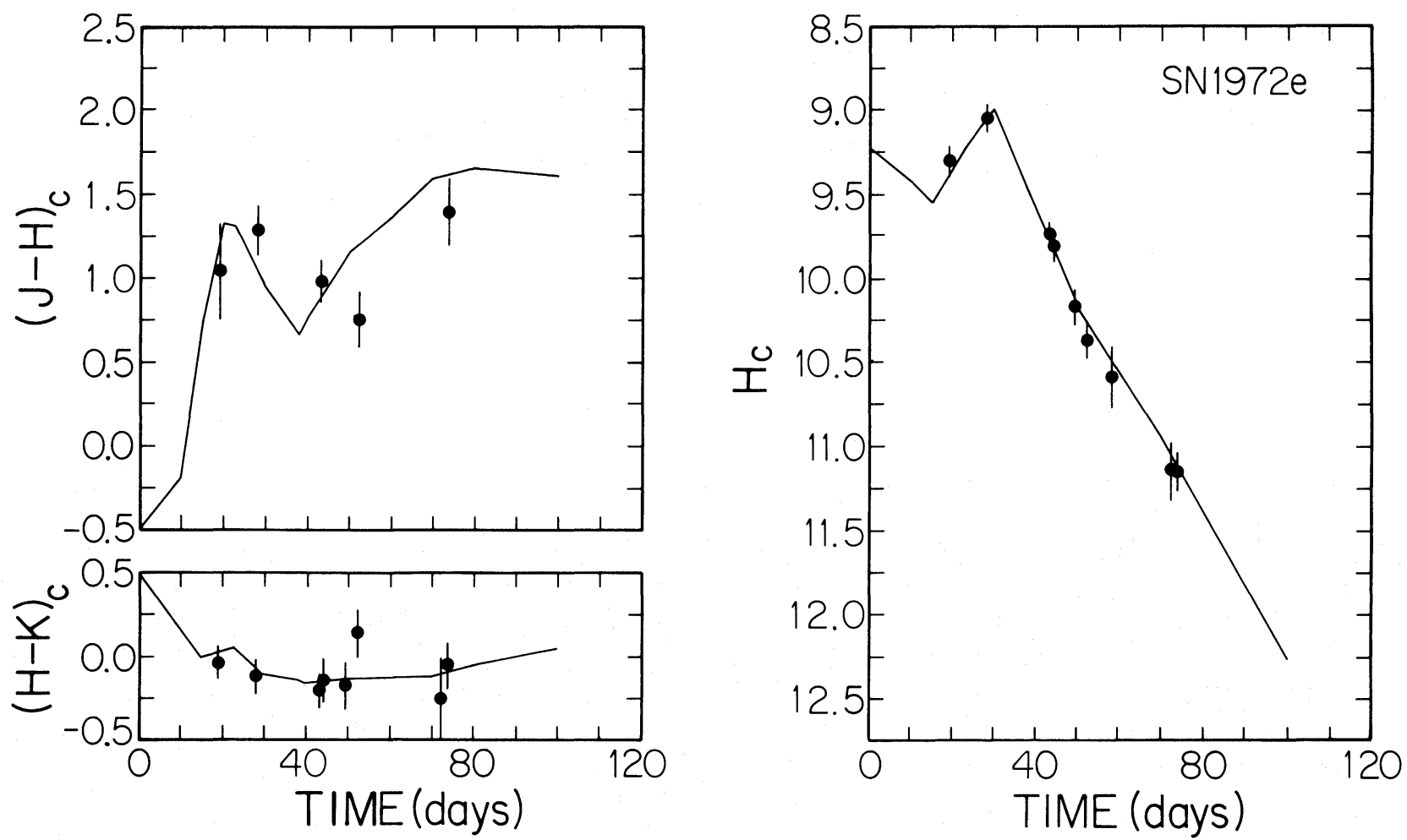

FIG. 3 - Infrared data for SN 1972e, plotted as in Fig. 1 

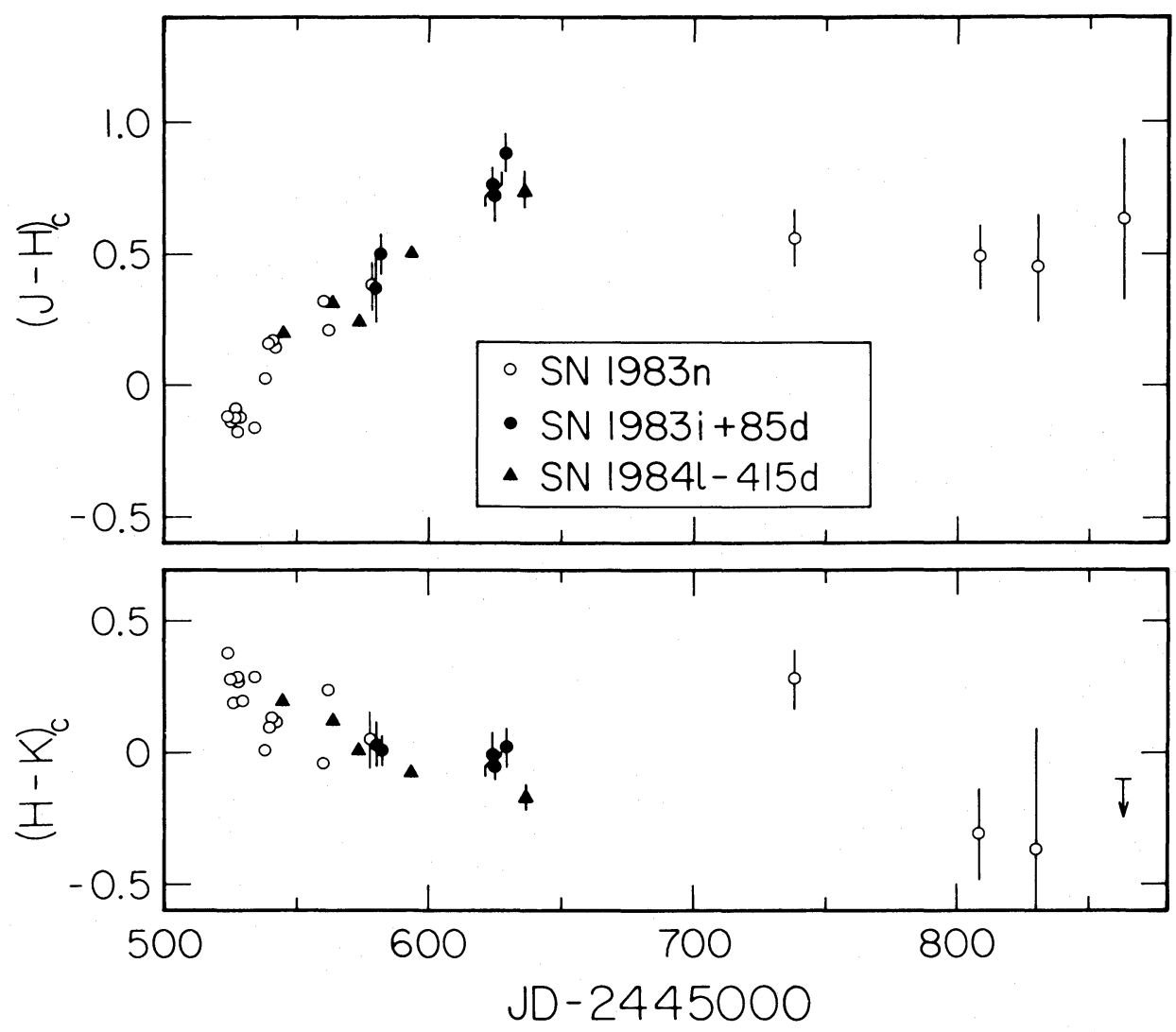

Fig. 4. $-J-H$ and $H-K$ color data for SN 1983i, SN 1983n, and SN 19841 plotted as in Fig. 1. Times were adjusted according to best estimate of time of visual maximum.

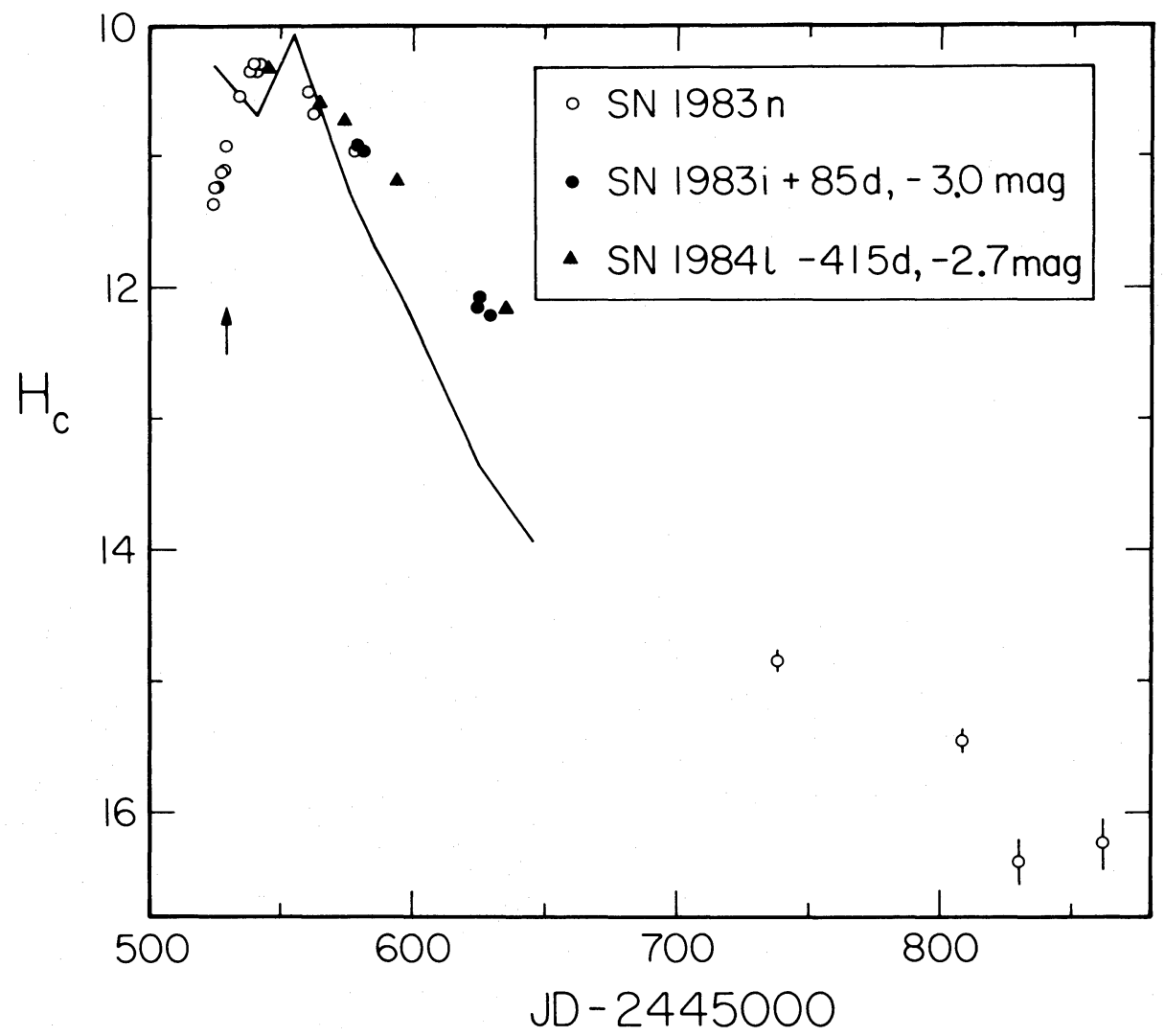

FIG. 5.- $H$ magnitude data for SN 1983i, SN 1983n, and SN 19841, plotted as in Fig. 1. Magnitudes were adjusted to give best fit to composite curve (see text). 
maximum is slower than the normal supernovae. If the double maximum structure of the normal light curves is caused by a variable absorption which is strongest at $1.2 \mu \mathrm{m}$ and present at 1.6 and $2.2 \mu \mathrm{m}$ (Paper I), then the simplest explanation of the differences between the two types of light curves may be that this absorption is absent in the Type $\mathrm{Ib}$ supernovae. There are no known, marked differences between the two classes of supernovae in the visual.

\section{TYPE Ia SUPERNOVAE AS DISTANCE INDICATORS}

Of the 13 Type I supernovae with infrared data, at least eight can be considered Type Ia. The evidence from Paper I indicated that the dispersion in their absolute magnitude near maximum is small, making them potentially useful distance indicators. We now reexamine this question with a larger data set.

The distances of the parent galaxies used for comparison were calculated using the galaxy redshifts and the linear infall model described by Aaronson et al. (1982) (using $V_{\mathrm{Vir}}=1019$ $\mathrm{km} \mathrm{s}^{-1}$ and $w_{c}=300 \mathrm{~km} \mathrm{~s}^{-1}$ ). This model was adopted because of its relative simplicity and its ability to provide distance estimates for all the galaxies in our sample. These distances are listed in Table 1. The apparent magnitudes of the individual supernovae, $\mathrm{H}_{20}$, are plotted against distance in Figure 6.

Two of the supernovae, SN 19711 in NGC 6384, and SN $1971 \mathrm{i}$ in NGC 5055, are not necessarily Type Ia. Three of the galaxies, NGC 4419, 4536, and 4753, lie in the general direction of Virgo, where the linear infall model is unreliable. The large blueshift of NGC 4419 and its position in the sky imply that it is near the center of the Virgo cluster, with a large peculiar velocity (Tully and Shaya 1984). For the other two galaxies, one cannot make so strong a case; NGC 4536 is likely to be no closer than $0.8 d_{\mathrm{Vir}}$; other considerations suggest that it is probably not much more distant, either (Buta and Turner 1983). NGC 4753 could be anywhere between about 0.6 and $1.5 d_{\mathrm{Vir}}$.
There are thus only six unambiguous Type Ia supernovae with well-determined distances. These define a relation with a scatter of $\sim 0.2 \mathrm{mag}$ which gives $H_{20}=13.1$ in the Virgo cluster. An increase in the adopted infall velocity $w_{i}$ to $350 \mathrm{~km}$ $\mathrm{s}^{-1}$ reduces the scatter to about $0.1 \mathrm{mag}$ because the low points in Figure 6 are all supernovae in the south Galactic hemisphere.

The value for SN 19711 in NGC 6384 is consistent with its being of Type Ia, but the value for SN $1971 \mathrm{i}$ is too faint. It could be an object similar to SN 1983 i in NGC 4051, which would also lie well above the mean relation, but which is demonstrably peculiar. The value for SN 1983g in NGC 4753 implies that the galaxy is at a distance of roughly $1.2 d_{\mathrm{Vir}}$.

A distance calibration where one is forced to adjust or reject such a large fraction of the data points is not overly reassuring. It is clear that one would like to double or triple the number of supernovae with good infrared data before setting out to use the calibration. It is also clear that any use of the calibration will require color data adequate to distinguish the two kinds of Type I supernovae from each other.

\section{3.5 MICRON FLUX}

The single marginal detection for $t=19$ days of SN 1983 in NGC 4753 at $3.7 \mu \mathrm{m}\left(L^{\prime}\right)$ is the best measurement at this wavelength of a Type Ia supernovae. Two good $3 \sigma$ upper limits exist-for SN 1980n in NGC 1316, where $K-L<+0.6$ at $t=38$ days (Paper I), and SN 1972e in NGC 5253, where $K-L<-0.2$ at $t=29$ days (Kirshner et al. 1973b; see Appendix). Other upper limits reported in Paper I, for SN $1981 \mathrm{~b}$ in NGC 4536 at $t=23$ days and for SN 1981d in NGC 1316 at $t=16$ days, are less constraining $(K-L<2)$. The NGC 4753 measurement corresponds to $K-L^{\prime} \approx-0.6_{-0.7}^{+0.4}$ or a $3 \sigma$ upper limit of $K-L^{\prime}<+0.4$. These three measurements individually allow $K-L$ (or $K-L^{\prime}$ ) to be close to zero, but collectively they suggest that $K-L$ around $t=30$ days is less than or equal to -0.5 . During this same time interval $J-H$ is

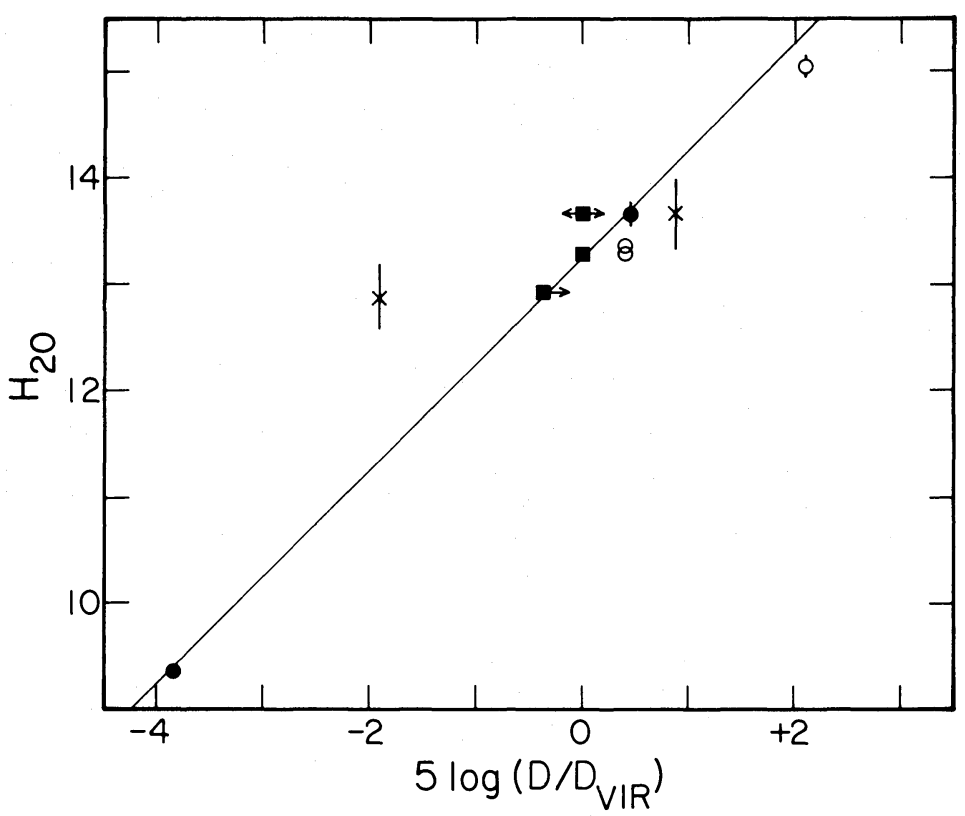

FIG. 6.-Plot of reference magnitude, $H_{20}$, against galaxy distance, $D / D_{\mathrm{Vir}}$. The straight line represents constant absolute magnitude. Open circles are galaxies in

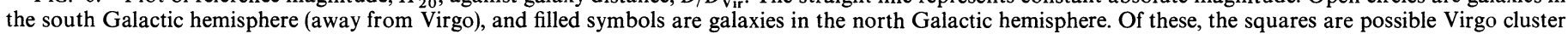
members. The crosses are supernovae with $K$ data only (see text for discussion). 


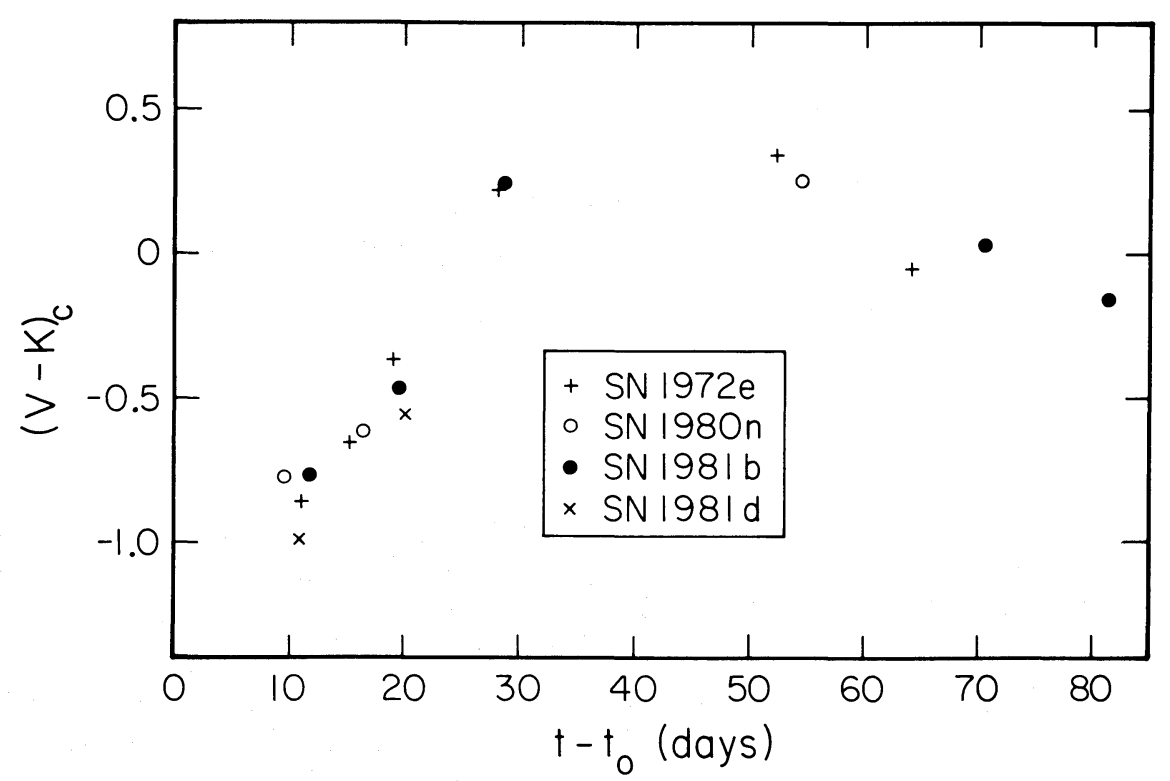

FIG. 7. $-V-K$, corrected for foreground extinction, plotted against time. Infrared data are from this paper and Paper I. Visual data are from references given in legend to Fig. 8 .

in the range +0.7 to +1.4 (Paper I), suggesting that there is a depression of the continuum at $\sim 3.5 \mu \mathrm{m}$ about half as large as the depression at $1.2 \mu \mathrm{m}$. Higher accuracy measurements at 3.5 $\mu \mathrm{m}$ near $t=20$ days are needed to confirm this.

The Type Ib SN 1983n in NGC 5236 was detected at $L$ and $L$ by Meikle et al. (1985), a few days after maximum light; the $K-L$ and $K-L^{\prime}$ colors are near zero. The measurement of SN 19841 in NGC 991 (Table 2) is consistent with a $K-L^{\prime}$ color near zero. If the Type Ia supernovae prove to have depressed $3.5 \mu \mathrm{m}$ flux, this would be yet another difference between the two types.

\section{CORRELATION WITH VISUAL DATA}

Five of the eight Type Ia supernovae have extensive visual photoelectric photometry, which can be used to see how uniform visual-infrared light curves are and to see how phases determined from $B-V$ colors agree with those determined from the infrared data.

$$
\text { a) } V-K
$$

There are four supernovae with enough overlap between the visual and infrared data to construct $V-K$ colors (SN 1972e, $1980 \mathrm{n}, 1981 \mathrm{~b}$, and $1981 \mathrm{~d})^{5}$; these are plotted, corrected for foreground extinction by $0.69 A_{B}$ in Figure 7 . The data are rather sparse, but it is clear that the $V-K$ color is never very red. Its evolution is similar to that of the $B-V$ color (Pskovskii 1977) - it starts out very blue, with $V-K \leq-0.8$, and gradually becomes redder, reaching $V-K \approx 0.4$ somewhat between $t=30$ days and $t=50$ days. Thereafter, $V-K$ slowly becomes bluer again. The $V-H$ color evolves in much the same way. Because of the transient $1.2 \mu \mathrm{m}$ depression, the $V-J$ color starts out near -1 . and becomes still bluer, reaching a value of about -2 near $t=20$ days. It then

\footnotetext{
${ }^{5}$ The data of Walker and Marino (1982) for SN 1981d have been corrected for galaxy flux by an amount corresponding to $V=14.91, B-V=0.85$, following Schweizer (1980). For the remaining data, such corrections have been made by the visual observers prior to publication or else appear to be well under $0.1 \mathrm{mag}$.
}

becomes redder, probably approaching 0 around $t=40$ days and then becomes blue again, approaching values of -1.5 . This demonstrates how substantial the strength of the depression at $1.2 \mu \mathrm{m}$ is.

It is also worth noting how small the scatter in $V-K$ is for the four supernovae - roughly $\pm 0.1 \mathrm{mag}$, which is not significantly greater than the observational uncertainties. This suggests that absorption in the parent galaxies of these supernovae is small. This is not necessarily the case for all Type I supernovae, as is shown by the data for SN 1983g in NGC 4753. The closest visual wavelength (Harris et al. 1983) and infrared observations are separated by five days, but they imply $V-K \approx 0$ at $t \approx 18$ days. The shift in $V-K$ required to match Figure 7 is $\sim 0.5 \mathrm{mag}$, implying an internal $A_{V}$ for the supernova of $\sim 0.55 \mathrm{mag}$. The values for $t_{0}$ and $H_{20}$ in Table 1 are changed by $\sim 1$ day and $0.05 \mathrm{mag}$, respectively, if the data are corrected for this extinction. As will be seen from the discussion of the $B-V$ colors, SN $1983 \mathrm{~g}$ may well have anomalous colors rather than high internal extinction. ${ }^{6}$

$$
\text { b) } B-V
$$

In Figure 8, plots of $B-V$, corrected for foreground extinction by $0.24 A_{B}$ are shown for the four supernovae discussed above. The data were restricted to photoelectric photometry. Times are relative to $t_{0}$, rather than $t_{\text {max }}$. Surprisingly, the four supernovae which have $V-K$ colors plotted in Figure 7 show equal or greater spread in $B-V$ than in $V-K$. This cannot be attributed to internal extinction, which is almost 3 times less, nor to measurement errors which ought also to be less. The data for SN 1983g deviate by even more. The internal extinction inferred from the $V-K$ data would considerably reduce the discrepancy but not eliminate it. Alternatively, the phasing in time could be adjusted. Harris et al. (1983) infer a

${ }^{6}$ Note added in manuscript.-Buta, Corwin, and Opal (1985) have published additional visual photometry of SN 1983g. These data show the $V-K$ color of this supernova to be close to those of the other objects in Fig. 7 after $t=30$ days, suggesting that the color anomalies are not produced by internal reddening by NGC 4753 . 


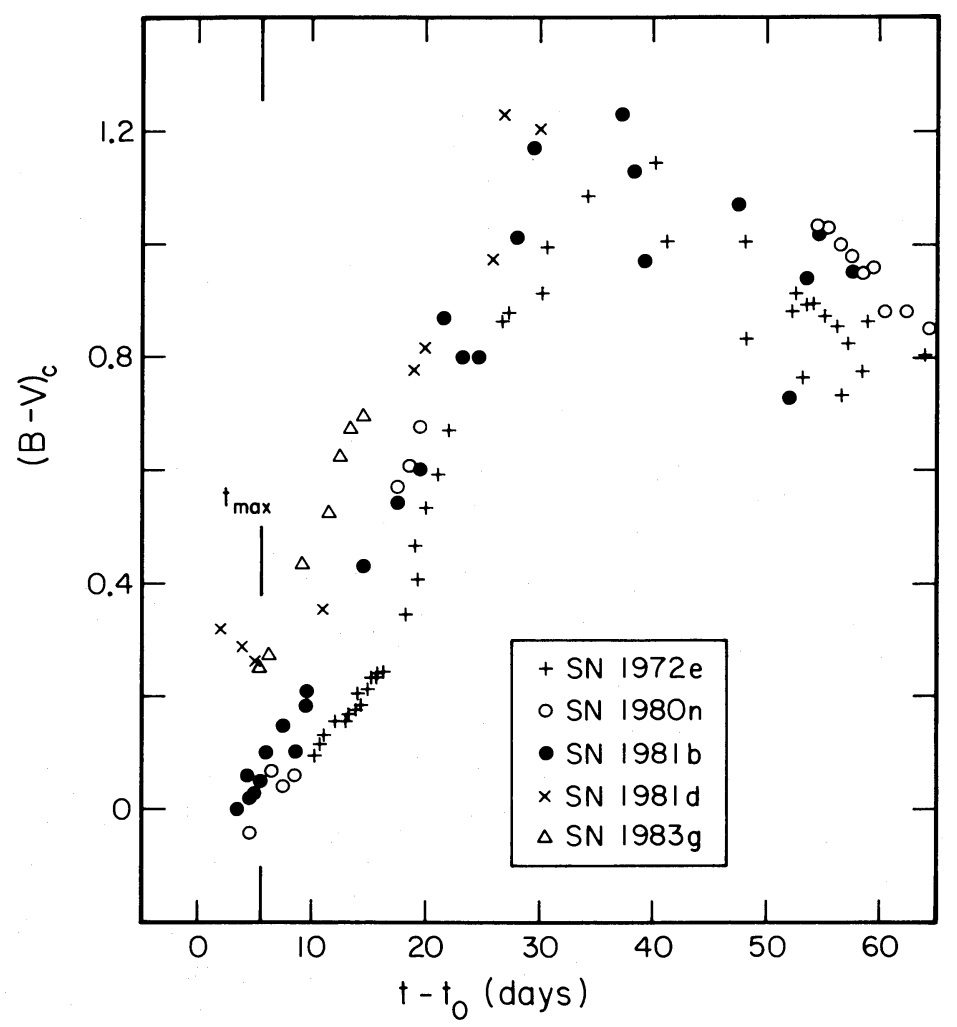

Fig. 8. $-B-V$, corrected for foreground extinction, plotted against time. Data for SN 1972e are from Lee et al. (1972), Ardeberg and de Groot (1973), and Eggen (1981). Data for SN 1980n are from Landolt $(1980,1984)$ and Olsiewski (1982). Data for SN 1981b are from Busko, Jablonski, and Torres (1981), Tsvetkov (1982), and Buta and Turner (1983). Data for SN 1981d are from Walker and Marino (1982). Data for SN 1983g are from Corwin (1983) and Harris et al. (1983).
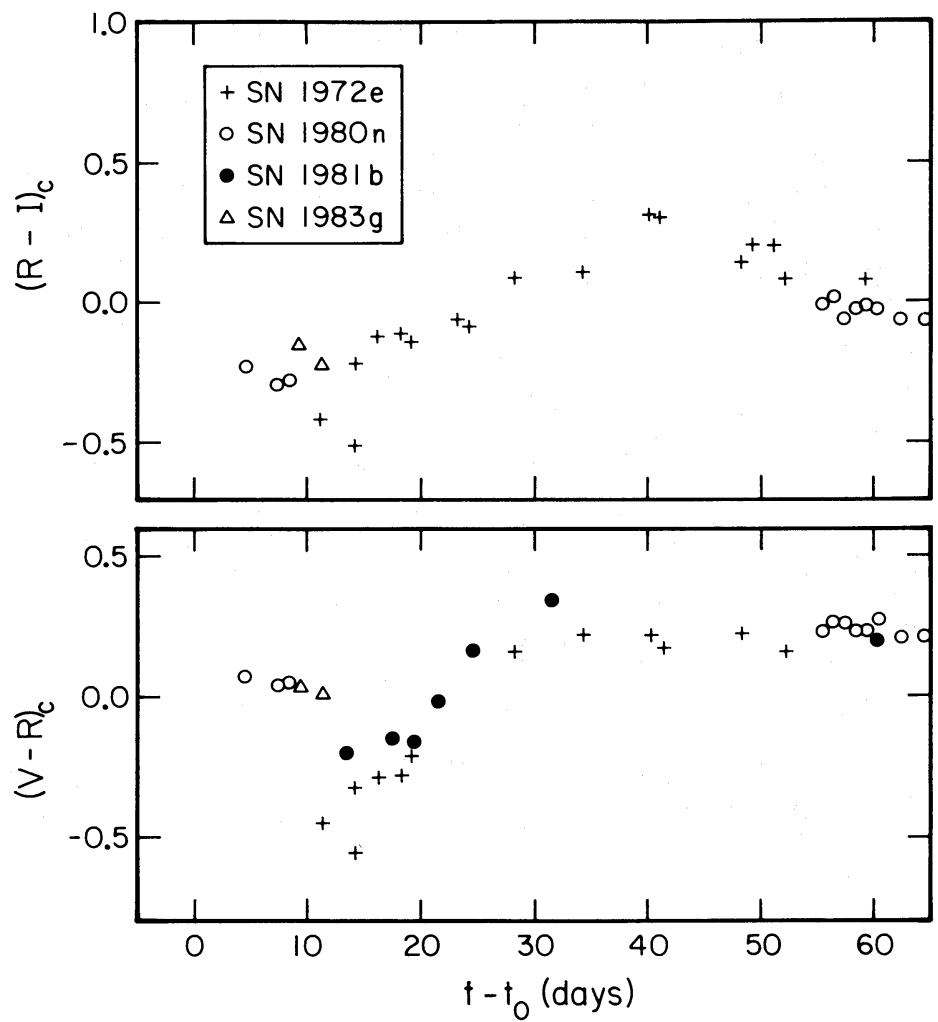

FIG. 9. $-V-R$ and $R-I$, corrected for foreground extinction plotted against time. Data sources are the same as for Fig. 8. 
time of maximum roughly 12 days earlier than that used in plotting Figure 8 ; this would bring the $B-V$ data into line but would produce severe discrepancies in the fit of the infrared data (Fig. 2). A careful comparison of the spectra given by Harris et al. with those presented by Kirshner et al. (1973a) and the descriptions of Minkowski (1939) supports the later time of maximum; the earliest spectra of $\mathrm{SN} 1983 \mathrm{~g}$ appear to be at most a few days after maximum. The spread among the $B-V$ color curves may indicate that $B-V$ colors of Type Ia supernovae have an intrinsic dispersion, which could be as large as $0.1 \mathrm{mag}$. Such dispersion would severely limit the accuracy of quantities traditionally deduced from $B-V$ colors, such as extinction or time of maximum.

\section{c) $V-R$ and $R-I$}

Supernova $V-R$ and $R-I$ colors are quite sparse. The available data have been plotted in Figure 9. All the points have been transformed to the Cousins system, using Bessell's (1979) transformations. These transformations are not very reliable for objects as peculiar as supernovae; undoubtedly, much of the scatter in Figure 9 is due to the variety of $V R I$ photometric systems in which the data were taken.

The evolution of the $V-R$ and $R-I$ colors shows that the strong $1.2 \mu \mathrm{m}$ depression extends weakly as far as the $R$ band; the $V-R$ color reaches a value near -0.3 mag around $t=15$ days. Both colors also show a gradual reddening trend with time, similar to that seen in $V-K$ and $B-V$.

It is also clear that the problem of the discrepant $B-V$ values for SN $1983 \mathrm{~g}$ discussed above cannot be cured by shifting the time of maximum - moving the points to the rightsince it would then make the $V-R$ colors anomalously red. Application of an internal reddening correction for this object is acceptable.

\section{IMPLICATIONS FOR PHOTOSPHERE MODELS}

The data presented for the five Type Ia supernovae show that a larger set of broad-band colors follows the same overall reddening with time described by Kirshner et al. (1973b). They also show that considerable caution must be used in inferring time of maximum from $B-V$ color data alone. Infrared photometry or visual spectrophotometry appear to provide more reliable and consistent estimates if maximum light was not actually observed.

It is clear that, given the discrepancies in the $B-V$ color curves and the broad depression in the red and infrared, use of a single color to determine effective temperature is unwise; a fit to the whole energy distribution, such as that performed by Kirshner et al. (1973b) or Branch et al. (1983) is clearly preferable. If an expansion parallax is determined (e.g., Branch et al. 1983), use of a complete energy distribution is clearly preferable to use of $B, V$ data only. Nevertheless, until supernova model atmospheres can provide an explanation for the $1.2 \mu \mathrm{m}$ absorption and its evolution, expansion parallaxes should be viewed with caution.

There are still few published visual data for the Type Ib supernova, although what there is (Meikle et al. 1985; Buta 1984) suggests that they are somewhat redder overall than Type Ia supernovae. Understanding of the differences between the two types of supernovae is probably critical to an understanding of the photospheres. A number of observations would be valuable in this regard. First, the overall energy distributions need to be compared, either by means of visual photometry to complement in the infrared data, or by absolute spectrophotometry in the visual. Second, the origin of the absorption at $1.2 \mu \mathrm{m}$ may be rendered less mysterious by infrared spectrophotometry.

\section{CONCLUSIONS}

The results of our program to date provide two new results. First, there are two subtypes of Type I supernova, which can be readily distinguished on the basis of their infrared color curves. The more common, Type Ia supernovae show a strong, variable absorption at $1.2 \mu \mathrm{m}$; the Type Ib supernovae do not.

Second, an examination of the use of infrared photometry of Type Ia supernovae for distance determinations suggests that distance moduli to individual galaxies may be accurate to $\pm 0.2 \mathrm{mag}$, and possibly $\pm 0.1 \mathrm{mag}$, but the data set is still too small for this conclusion to be independent of our initial assumptions.

Further results concern the evolution in color of the Type Ia supernovae. There is evidence for an absorption feature at 3.5 $\mu \mathrm{m}$, possibly similar in its behavior to the feature at $1.2 \mu \mathrm{m}$, although probably not as strong.

The $V-K$ colors evolve much like visual colors, starting out rather blue, and becoming redder with time for $\sim 40$ days, and then becoming slightly bluer with time.

The $B-V$ color may have an intrinsic dispersion of perhaps $0.1 \mathrm{mag}$. The individual colors deviate from those due to a simple blackbody, sometimes grossly so, and this suggests that temperatures inferred from either fits to single colors or even to the energy distribution as a whole must be regarded as approximate at best.

We would like to thank R. P. Kirshner and S. P. Willner for helpful conversations and aid in recovering the SN 1972e data. We would also like to thank B. Madore, C. McAlary, M. Aaronson, and J. Mould for obtaining two of the observations in Table 1. We would also like to thank O. J. Eggen and A. U. Landolt for providing their unpublished visual photometry on SN 1972e and SN 1980n. This work has been supported by the NSF under grant AST 83-12699.

\section{APPENDIX}

\section{SN 1972e DATA}

The data presented by Kirshner et al. (1973b), for SN 1972e, as well as later observations, are summarized in Table 4. In several cases, the data have been reduced again, using improved values for standard star magnitudes. There has been no correction for the underlying galaxy; the size of this correction for small-beam measurements is probably well indicated by the last three measurements in the table. These results indicate that corrections to the earlier large telescope measurements are probably roughly $1 \%$. For the $0.6 \mathrm{~m}$ telescope measurements, the corrections could plausibly be several times larger, although examination of the data indicates that the systematic effects are less than $0.1 \mathrm{mag}$. Since all the color information was taken with the larger telescopes, and 
TABLE 4

ARChival SUPERNOVA PHOTOMETRY OF SN 1972e

\begin{tabular}{|c|c|c|c|c|c|c|}
\hline \multirow{2}{*}{$\begin{array}{c}\text { JD - } \\
2,440,000\end{array}$} & \multicolumn{4}{|c|}{ OBSERVED MAGNITUDES $^{\mathrm{a}}$} & \multirow[b]{2}{*}{ TELESCOPE $^{\mathrm{b}}$} & \multirow{2}{*}{$\begin{array}{l}\text { APERTURE } \\
\text { (arcsec) }\end{array}$} \\
\hline & $J$ & $H$ & $K$ & $L$ & & \\
\hline $1454.8 .$. & $\ldots$ & $\ldots$ & $9.41(21)$ & $\ldots$ & W24 & 60 \\
\hline $1458.8 \ldots \ldots$ & $\ldots$ & $\ldots$ & $9.48(25)$ & $\ldots$ & W24 & 60 \\
\hline $1459.7 \ldots \ldots$ & $\ldots$ & $\ldots$ & $9.44(20)$ & $\ldots$ & W24 & 60 \\
\hline $1461.8 \ldots \ldots$ & & & $9.29(16)$ & $\ldots$ & W24 & 60 \\
\hline $1463.6 \ldots \ldots$ & $10.38(26)$ & $9.32(8)$ & $9.35(8)$ & $\ldots$ & P200 & 15 \\
\hline $1465.7 \ldots \ldots$ & $\ldots$ & $\ldots$ & $10.88(185)^{\mathfrak{c}}$ & $\ldots$ & W24 & 60 \\
\hline $1468.7 \ldots \ldots$ & & & $9.39(27)$ & $\ldots$ & W24 & 60 \\
\hline $1472.6 \ldots \ldots$ & $10.37(12)$ & $9.07(8)$ & $9.18(8)$ & $\ldots$ & P200 & 15 \\
\hline $1473.6 \ldots \ldots$ & $\ldots$ & $\ldots$ & & $12.53(648)^{\mathrm{c}}$ & P200 & 10 \\
\hline $1477.7 \ldots \ldots$ & $\ldots$ & $\ldots$ & $10.03(29)$ & $\ldots$ & W24 & 60 \\
\hline $1480.8 \ldots \ldots$ & $\ldots$ & $\ldots$ & $9.56(24)$ & $\ldots$ & W24 & 60 \\
\hline $1482.8 \ldots \ldots$ & $\ldots$ & $\ldots$ & $9.00(16)$ & $\ldots$ & W24 & 60 \\
\hline $1484.7 \ldots \ldots$ & & & $10.12(32)$ & $\ldots$ & W24 & 60 \\
\hline $1487.7 \ldots \ldots$ & $10.76(10)$ & $9.76(8)$ & $9.95(8)$ & $* 10.75(-429)^{\mathrm{d}}$ & P200 & 7.5 \\
\hline $1488.7 \ldots \ldots$ & $\ldots$ & $9.83(10)$ & $9.96(9)$ & $\ldots$ & P200 & 7.5 \\
\hline $1493.7 \ldots \ldots$ & & $10.19(10)$ & $10.35(10)$ & $\ldots$ & W100 & 20 \\
\hline $1496.7 \ldots \ldots$ & $11.16(13)$ & $10.39(10)$ & $10.24(11)$ & $\ldots$ & P200 & 10 \\
\hline $1502.7 \ldots \ldots$ & $13.07(150)^{c}$ & $10.61(17)$ & $11.11(71)^{c}$ & $\ldots$ & W100 & 15 \\
\hline $1516.7 \ldots \ldots$ & $12.59(66)^{\mathrm{c}}$ & $11.16(16)$ & $11.40(17)$ & $\ldots$ & P200 & 7.5 \\
\hline $1517.7 \ldots \ldots$ & & $11.17(11)$ & $11.29(8)$ & $\ldots$ & P200 & 14 \\
\hline $1518.7 \ldots \ldots$ & $12.58(16)$ & $\ldots$ & $11.03(13)$ & $\ldots$ & P200 & 14 \\
\hline $1570.5 \ldots \ldots$ & $\ldots$ & $\ldots$ & $12.09(152)^{\mathrm{c}}$ & $\ldots$ & $\mathrm{C} 40$ & 30 \\
\hline $1707.1 \ldots \ldots$ & $\ldots$ & $\ldots$ & $12.18(30)$ & $\ldots$ & P200 & 7.5 \\
\hline $1708.0 \ldots \ldots$ & $\ldots$ & $\ldots$ & $15.13(153)^{\mathrm{c}}$ & $\ldots$ & P200 & 5 \\
\hline $1841.7 \ldots \ldots$ & $\ldots$ & $\ldots$ & $15.67(192)^{c}$ & $\ldots$ & P200 & 5 \\
\hline $2468.1 \ldots \ldots$ & $\ldots$ & $\ldots$ & $16.47(71)^{\mathrm{c}}$ & $\ldots$ & P200 & 10 \\
\hline
\end{tabular}

${ }^{\text {a }}$ Percent errors are given in parentheses.

b Telescope codes are P200 = Palomar $5 \mathrm{~m}$; W100 = Mount Wilson $2.5 \mathrm{~m}$; W24 = Mount Wilson $0.6 \mathrm{~m}$; and $\mathrm{C} 40=$ Las Campanas $1.0 \mathrm{~m}$. Beam spacing was usually in declination with typical values of $15^{\prime \prime}, 30^{\prime \prime}$, $120^{\prime \prime}$, and $90^{\prime \prime}$ for the respective telescopes.

c Not a significant measurement $(<3 \sigma)$.

d Not a significant measurement; nominal value represents a negative flux equivalent to $10.75 \mathrm{mag}$.

since the $0.6 \mathrm{~m}$ measurements are of lower accuracy (typically $20 \%$ ), the fitting of the light curves was done using only the 10 measurements from the $2.5 \mathrm{~m}$ and $5 \mathrm{~m}$ telescopes. The measurements on JD 1517.7 and 1518.7 were averaged in order to produce a $J-H$ color.

\section{REFERENCES}

Aaronson, M., Huchra, J., Mould, J., Schechter, P. L., and Tully, R. B. 1982, Ap. J., 258, 64 .

Ardeberg, A., and de Groot, M. 1983, Astr. Ap., 28, 295.

Barbon, R. 1980, in Proc. Texas Workshop on Type I Supernovae, ed. J. C. Wheeler (Austin: University of Texas), p. 16.

Bessell, M. S. 1979, Pub. A.S.P., 91, 589.

Branch, D., Lacy, L. H., McCall, M. L., Sutherland, P. G., Uomoto, A.

Wheeler, J. C., and Wills, B. J. 1983, Ap. J., 270, 123.

Burstein, D., and Heiles, C. 1984, Ap. J. Suppl., 54, 33.

Busko, I., Jablonski, F., and Torres, C. 1981, IAU Circ., 3589.

Buta, R. 1984, I AU Circ., 2891.

Buta, R., Corwin, H. G.,Jr., and Opal, C. B. 1985, Pub. A.S.P., 97, 229.

Buta, R. J., and Turner, A. 1983, Pub. A.S.P., 95, 72.

Corwin, H. G., Jr. 1983, I AU Circ., 3794.

de Vaucouleurs, G. 1975, in Stars and Stellar Systems, Vol. 9, Galaxies and the Universe, ed. A. Sandage, M. Sandage, and J. Kristian (Chicago: University of Chicago Press), p. 557.

Doroshenko and Tsvetkov. 1983, IAU Circ., 3813.

Eggen, O. J. 1981, private communication.

Elias, J. H.; Frogel, J. A., Hackwell, J. A., and Persson, S. E. 1981, Ap. J. (Letters), 251, L13 (Paper I).

Graham, J. R., Geballe, T. R., Meikle, W. P. S., Andrews, P. L., Longmore, A. J., Williams, P. M., and Allen, D. A. 1984, IAU Circ., 4003.

Harris, G. J. H., Hesser, J. E., Massey, P., Peterson, C. J., and Yamanaka, J. 1983, Pub. A.S.P., 95, 607.
Kirshner, R. P., Oke, J. B., Penston, M. V., and Searle, L. 1973a, Ap. J., 185, 303.

Kirshner, R. P., Willner, S. P., Becklin, E. E., Neugebauer, G., and Oke, J. B. 1973b, Ap. J. (Letters), 180, L97.

Landolt, A. U. 1980, IAU Circ., 3556.

.1984, private communication.

Lee, T. A., Wamsteker, W., Wiśniewski, W. Z., and Wdowiak, T. J. 1972, Ap. J. (Letters), 177, L59.

Marsden, B. 1984, IAU Circ., 4019

Meikle, W. P. S., Graham, J. R., and Bode, M. F. 1984, I AU Circ., 3918.

Meikle, W. P. S., and Walther, D. M. 1984, IAU Circ., 3924.

Meikle, W. P. S., et al. 1985 , in preparation.

Minkowski, R. 1939, Ap. J, 89, 143.

Olsiewski, E. W. 1982, Inf. Bull. Var. Stars, 2065.

Pskovskii, Yu. P. 1977, Soviet Astr., 21, 675.

Sandage, A., and Tammann, G. A. 1982, Ap. J., 256, 339.

Schweizer, F. 1980, Ap. J., 237, 303.

Tsvetkov, D. Yu. 1982, Pis'ma Astr. Zh., 8, 219.

Tully, R. B., and Shaya, E. J. 1984, Ap. J., 281, 31.

Walker, W. S. G., and Marino, B. F. 1982, Pub. Variable Star Section, Royal Astr. Soc., New Zealand, No. 10, p. 53.

Wheeler, J. C., and Levreault, R. 1985, Ap. J. (Letters), 294, L17.

J. H. Elias, K. Matthews, and G. Neugebauer: California Institute of Technology, Downs Laboratory of Physics 320-47, Pasadena, CA 91125

S. E. Persson: Mount Wilson and Las Campanas Observatories, 813 Santa Barbara Street, Pasadena, CA 91101 